\title{
Unsupervised Machine and Deep Learning Methods for Structural Damage Detection: A Comparative Study
}

\author{
Zilong Wang ${ }^{1}$ and Young-Jin Cha ${ }^{2}$ \\ ${ }^{1}$ Suzhou Institute of Building Science Group \\ ${ }^{2}$ University of Manitoba Faculty of Engineering
}

February 25, 2022

\begin{abstract}
While many structural damage detection methods have been developed in recent decades, few data-driven methods in unsupervised learning mode have been developed to solve the practical difficulties in data acquisition for civil infrastructures in different scenarios. To address such a challenge, this paper proposes a number of improved unsupervised novelty detection methods and conducts extensive comparative studies on a laboratory scale steel bridge to examine their performances of damage detection. The key concept behind unsupervised novelty detection in this paper is that only normal data from undamaged structural scenarios are required to train statistical models with these methods. Then, these trained models are used to identify abnormal testing data from damaged scenarios. To detect structural damage in the form of loosening bolts in the steel bridge, four machine-learning methods (i.e., K-nearest neighbors method, Gaussian mixture models, One-class support vector machines, Density peaks-based fast clustering method) and one deep learning method using a deep auto-encoder are selected. Meanwhile, some modifications and improvements are made to enable these methods to detect structural damage in unsupervised novelty detection mode. In their comparative studies, the advantages and disadvantages of these methods are analyzed based on their results of structural damage detection.
\end{abstract}

\section{Hosted file}

Manuscript_2022-02-21_final.docx available at https://authorea.com/users/462278/articles/ 557746-unsupervised-machine-and-deep-learning-methods-for-structural-damage-detection-acomparative-study 